Zbl Arbeitsmed $2021 \cdot 71: 281-287$ https://doi.org/10.1007/s40664-021-00443-9 Eingegangen: 30. März 2021

Überarbeitet: 9. August 2021

Angenommen: 11. August 2021

Online publiziert: 23 . September 2021

(c) Der/die Autor(en) 2021
Peter Koch ${ }^{1}$ Gabriele Halsen ${ }^{2}$. Lukas Damerau ${ }^{3}$ Albert Nienhaus ${ }^{1,2}$. Volker Harth ${ }^{3}$. Matthias Augustin ${ }^{1}$. Claudia Terschüren ${ }^{3}$

'Institut für Versorgungsforschung in der Dermatologie und bei Pflegeberufen, Universitätsklinikum Hamburg-Eppendorf (UKE), Hamburg, Deutschland

${ }^{2}$ Abteilung Arbeitsmedizin, Gefahrstoffe und Gesundheitswissenschaften (AGG), Berufsgenossenschaft für Gesundheitsdienst und Wohlfahrtspflege (BGW), Hamburg, Deutschland

${ }^{3}$ Zentralinstitut für Arbeitsmedizin und Maritime Medizin (ZfAM), Universitätsklinikum HamburgEppendorf (UKE), Hamburg, Deutschland

\title{
Wissen über berufsbedingte inhalative Noxen bei Beschäftigten im Gesundheitsdienst und der Wohlfahrtspflege
}

\section{Hintergrund und Fragestellung}

Beruflich bedingte inhalative Noxen (Dämpfe, Gase, Stäube, Rauche) sind vor allem an Industriearbeitsplätzen vorzufinden. Ebenso finden sich aber inhalative Noxen bei Beschäftigten im Gesundheitsdienst und der Wohlfahrtspflege wieder. In den verschiedenen Tätigkeitsfeldern dieser Branche existieren insbesondere verschiedene chemische Gefahrstoffgruppen, die potenziell von Beschäftigten inhalativ aufgenommen werden können. Dazu gehören Arzneimittel, Desinfektionsmittel und -reiniger, Inhalationsanästhetika, spezielle Gefahrstoffe in der Zahnmedizin, Chemikalien im Labor und Röntgen, Sterilisationsgase und -dämpfe, medizinische Gase sowie chirurgische Rauchgase [12].

Die oberen und unteren Atemwege stellen die ersten Kontaktorgane gegenüber inhalativen Noxen dar. Die Gesundheitsgefahren bei Inhalation können akute Toxizität, Sensibilisierung der Atemwege, und Karzinogenität sein. Zu den berufsbedingten obstruktiven Atemwegserkrankungen zählen das Asthma bronchiale, die chronisch-obstruktive Lungenerkrankung (COPD), das Lungenemphysem und die chronische Bronchitis. Studien zeigen, dass
15-31\% aller COPD-Fälle auf eine berufliche Exposition zurückgeführt werden können $[1,13,17]$. Der populationsattributable Anteil beruflicher Faktoren für Asthma liegt zwischen 10 und $15 \%$ [3, $21,28]$. Für das Lungenkarzinom liegen die Schätzungen des Anteils beruflichen Einflusses zwischen 10 und $33 \%$ vor [2, 20].

Zur Prävention im berufsbezogenen Umgang mit inhalativen Gefahrstoffen gibt es an oberster Stelle das deutsche Arbeitsschutzgesetz bzw. die europäische Rahmenrichtlinie zur Sicherheit und Gesundheitsschutz bei der Arbeit [7, 8]. Diese Vorschriften sollen das Wissen dazu liefern und verteilen, um diese Gesundheitsgefahren am Arbeitsplatz frühzeitig zu erkennen und wirkungsvolle Schutzmaßnahmen einzuleiten. Die Adressaten für diese Informationen sind die Unternehmensleitungen sowie die Beschäftigten.

Unseres Wissens gibt es bislang keine Untersuchungen, die das Wissen über berufsbedingte inhalative Noxen bei Beschäftigten im Gesundheitsdienst und der Wohlfahrtspflege in Deutschland untersucht haben.

In der vorliegenden Untersuchung soll anhand einer bevölkerungsbezogenen Stichprobe analysiert werden, inwiefern Beschäftigte aus dem Gesundheitsdienst und der Wohlfahrtspflege sich über inhalative Noxen an ihrem Arbeitsplatz bewusst sind.

\section{Methoden}

Grundlage zur Ermittlung der Prävalenz des individuellen Expositionswissens liefern Daten der Baseline-Stichprobe der Hamburg City Health Studie (HCHS; [19]). Die HCHS ist eine bevölkerungsbezogene epidemiologische Kohortenstudie, die im Zeitraum 2017-2019 über das Stichprobenverfahren Baselinedaten von 10.000 Probanden im Alter von 45-74 Jahren aus Hamburg erhoben hat. Das primäre Ziel dieser großangelegten Studie ist die Ermittlung von Risikofaktoren für Herz-Kreislauf-Erkrankungen und Demenz im Längsschnitt.

\section{Fragebogen}

Neben demografischen Angaben (Alter, Geschlecht, Geburtsland, Schulabschluss, Erwerbsstatus) sind Daten zur Berufsbiografie extrahiert worden. Die aktuelle bzw. zuletzt ausgeübte berufliche Tätigkeit wurde anhand von drei Freitextangaben erhoben: 


\begin{tabular}{|c|c|c|c|c|}
\hline & $\begin{array}{l}\text { Weiblich } \\
916 \text { (77,9\%) }\end{array}$ & $\begin{array}{l}\text { Männlich } \\
260(22,1 \%)\end{array}$ & $\begin{array}{l}\text { Gesamt } \\
1176(100 \%)\end{array}$ & $p$-Wert \\
\hline \multicolumn{4}{|l|}{ Alter (Jahre) } & $<0,001^{\mathrm{a}}$ \\
\hline MW (SD) & $60,4(7,8)$ & $62,2(8,4)$ & $60,8(8,0)$ & - \\
\hline Spannweite & $46,0-78,0$ & $46,0-78,0$ & $46,0-78,0$ & - \\
\hline \multicolumn{4}{|l|}{ Geburtsland } & $0,024^{b}$ \\
\hline Deutschland & $755(84,2 \%)$ & $229(89,8 \%)$ & $984(85,4 \%)$ & - \\
\hline Anderes & $142(15,8 \%)$ & $26(10,2 \%)$ & $168(14,6 \%)$ & - \\
\hline$n$ fehlend & 19 & 5 & 24 & - \\
\hline \multicolumn{4}{|l|}{ Schulabschluss } & $<0,001^{b}$ \\
\hline Hoch & $428(47,9 \%)$ & $191(74,9 \%)$ & $619(53,9 \%)$ & - \\
\hline Mittel & $299(33,4 \%)$ & $39(15,3 \%)$ & $338(29,4 \%)$ & - \\
\hline Niedrig & $148(16,6 \%)$ & $22(8,6 \%)$ & $170(14,8 \%)$ & - \\
\hline Keinen & $19(2,1 \%)$ & $3(1,2 \%)$ & $22(1,9 \%)$ & - \\
\hline$n$ fehlend & 22 & 5 & 27 & - \\
\hline \multicolumn{4}{|l|}{ Arbeitsstatus } & $0,484^{b}$ \\
\hline Erwerbstätig & $609(68,9 \%)$ & $178(71,2 \%)$ & $787(69,4 \%)$ & - \\
\hline Nichterwerbstätig & $275(31,1 \%)$ & $72(28,8 \%)$ & $347(30,6 \%)$ & - \\
\hline$n$ fehlend & 32 & 10 & 42 & - \\
\hline \multicolumn{5}{|c|}{$\begin{array}{l}\text { aMann-Whitney-U-Test } \\
\text { 'Pearson's Chi'-Test }\end{array}$} \\
\hline
\end{tabular}

1. Berufsbezeichnung,

2. Beschreibung der beruflichen Tätigkeit,

3. Branche des Betriebes.

Weiterhin wurde zum Vorhandensein über inhalative Noxen am Arbeitsplatz folgende Frage gestellt: Haben Sie jemals an einem Arbeitsplatz gearbeitet, an dem Sie Dämpfen, Gasen, Stäuben/Staub oder Rauch ausgesetzt waren? Also möglicherweise Gefahrstoffe eingeatmet haben (ja/nein/weiß nicht/keine Angabe)? Nachgeordnet zu dieser Filterfrage im Eingangsfragebogen war jeweils zu jeder einzelnen Expositionsart (Dämpfe, Gase, Stäube/Staub, Rauch) eine Frage zu der Häufigkeit der Exposition am Arbeitsplatz gestellt (nie/selten/häufig/immer/ weiß nicht). Aufgrund eines sehr hohen Anteils fehlender Angaben (80-90\%), die im nachgeordneten dritten Fragebogen (nach dem Untersuchungstag im Studienzentrum) erfasst wurden, wurde die selbstberichtete Häufigkeit nach Expositionsart in dieser Analyse nicht berücksichtigt.

\section{Expositionsdaten anhand einer Job-Exposure-Matrix (JEM)}

Für den Abgleich mit den subjektiven Expositionsdaten aus dem Fragebogen ist das Instrument der Job-Exposure-Matrix (JEM) ausgewählt worden. Beim Einsatz einer JEM können auch denjenigen Personen einer Exposition zugewiesen werden, die sich ihrer Exposition am Arbeitsplatz nicht bewusst sind. Nach einer umfangreichen Recherche zu verschieden JEM wurde dazu die „airborne chemical exposure job-exposure matrix" (ACE JEM) ausgewählt, die spezifisch für Atemwegserkrankungen entwickelt wurde [26]. Die ACE JEM verzeichnet Expositionen gegenüber Dämpfen, Gasen, Stäuben und Rauchen differenziert nach vier Expositionshöhen (keine/ wenig/mittel/hoch). Die Expositionszuordnung der JEM zeigt für die Berufsgruppen, in denen die betrachteten Expositionsstoffe zwar im Arbeitsumfeld vorhanden sind, aber kein primäres Arbeitsmittel darstellen bzw. wenig genutzt der jeweiligen Berufsgruppe der Expositionshöhe "wenig" zugeordnet wird. Dies trifft für Beschäftigte in der Krankenpflege/Rettungsdienst/Geburtshilfe werden, dass zwischen 90 und $100 \%$ und der Altenpflege sowie für Mediziner und Tiermediziner zu. Weiterhin zeigen die Zuordnungen in der JEM, dass für Berufe, bei denen man davon ausgehen kann, dass die Substanzen der untersuchten Exposition Bestandteil eines Hauptarbeitsmittels sind, dass mindestens $80 \%$ der jeweiligen Berufsgruppe der Expositionshöhe "mittel“ bzw. "hoch“" zugeordnet sind. Es wurden zwei Varianten ausgewertet: Neben der Kategorisierung (I) Nichtexponierte: keine Exposition und Exponierte: wenig/ mittel/hohe Exposition wurde auch mit einer alternativen Expositionskategorisierung (II) gerechnet: Nichtexponierte: keine/wenig Exposition und Exponierte: mittel/hohe Exposition.

\section{Zuordnung der JEM-Expositions- daten zu den Probandendaten}

Anhand der drei selbstberichteten Freitextangaben zur beruflichen Tätigkeit (Branche, Beruf, Tätigkeit) im Fragebogen erfolgte eine Klassifikation in einen 5-stelligen Berufscode der deutschen Klassifikation der Berufe 2010 (KldB 2010) [6]. Anschließend erfolgte eine Übertragung in die International Standard Classification of Occupations 2008 (ISCO-08; [15]) und später in die britische Standard Occupational Classification 2000 (SOC2000; [14]). Diese Klassifikation ermöglichte es, den Berufscode des deutschen Klassifikationssystems mit den Daten der ACE JEM zu verlinken.

\section{Statistische Auswertung}

Die Präsentation der Ergebnisse erfolgt in absoluten und relativen Häufigkeiten. Bei Vergleichen von nominal bzw. kategorial skalierten Variablen wurde der $\mathrm{Chi}^{2}$ Test, bei Mittelwertsvergleichen wurde Fishers T-Test durchgeführt. Zur Berechnung der Übereinstimmung von zwei nominalen Variablen wurde Cohens Kappa berechnet. Die statistische Auswertung erfolgte mit SPSS 27.0.

\section{Ergebnisse}

Von 10.000 Probanden der HCHS konnte für 8171 Personen anhand der Freitext- 
Zbl Arbeitsmed $2021 \cdot 71: 281-287$ https://doi.org/10.1007/s40664-021-00443-9

(c) Der/die Autor(en) 2021

P. Koch · G. Halsen · L. Damerau · A. Nienhaus · V. Harth · M. Augustin · C. Terschüren

\section{Wissen über berufsbedingte inhalative Noxen bei Beschäftigten im Gesundheitsdienst und der Wohlfahrtspflege}

\section{Zusammenfassung}

Hintergrund. Das Einatmen von beruflich bedingten Noxen (Dämpfe, Gase, Stäube, Rauche) kann zu unterschiedlichsten Gesundheitsproblemen führen. Beschäftigte aus dem Gesundheitsdienst und der Wohlfahrtspflege sind bei ihrer Tätigkeit z. B. Inhalationsanästhetika, Desinfektionsmitteln und -reinigern und chirurgischen Rauchgasen ausgesetzt.

Fragestellung. In dieser Untersuchung soll ermittelt werden, inwieweit sich Beschäftigte im Gesundheitsdienst und der Wohlfahrtspflege ihrer berufsbedingten Exposition gegenüber inhalativen Noxen bewusst sind.

Material und Methoden. Aus der 10.000er Baseline-Stichprobe der HCHS (Hamburg City Health Study) wurden alle Probanden selektiert, die beruflich im Gesundheitsdienst und der Wohlfahrtspflege tätig waren. Die subjektiven Fragebogenangaben zu einer arbeitsplatzbezogenen Exposition von Dämpfen, Gasen, Stäuben und Rauchen wurden mit Daten einer Job-Exposure-Matrix (JEM) abgeglichen.

Ergebnisse. Die Stichprobe $(N=1176)$ bestand zu $78 \%$ aus Frauen, das Durchschnittsalter lag bei 61 Jahren. Probanden, die in der Arztund Praxishilfe $(60,5 \%)$ beschäftigt waren, machten die größte Berufsgruppe aus. Die Übereinstimmung zwischen subjektiven Expositionsangaben und JEM war gering (Cohens Kappa: 0,18). Die Sensitivität der subjektiven Angaben zu inhalativen Noxen im Vergleich zur JEM lag bei 49,5\%, die Spezifität bei $81,7 \%$. Ein Underreporting zu inhalativen Noxen war ausschließlich bei Beschäftigten in der Reinigung und der Körperpflege zu beobachten.
Diskussion. Die Ergebnisse zeigen, dass rund $50 \%$ der exponierten Beschäftigten dieser Stichprobe sich ihrer Exposition nicht bewusst sind. Dies betrifft speziell Beschäftigte in der Reinigung und der Körperpflege. Das Underreporting von inhalativen Noxen am Arbeitsplatz gibt einen Hinweis für einen erhöhten Präventionsbedarf in dieser Branche. Demgegenüber stehen die existierenden Regeln und Vorschriften des Arbeitsschutzsystems. Abzuklären bleibt, inwieweit Wissensvermittlung sowie Präventionsregeln hinsichtlich inhalativer Noxen am Arbeitsplatz in Betrieben tatsächlich umgesetzt werden.

\section{Schlüsselwörter}

Berufliche Tätigkeit - Luftschadstoffe . Sensitivität und Spezifizität · Prävention . Gesundheits- und Wohlfahrtspflege

\section{Knowledge of occupational inhalative noxious agents among healthcare and welfare workers}

\section{Abstract}

Background. Inhalation of occupation-related noxious agents (vapors, gases, dusts, fumes) can lead to a wide variety of health problems. Healthcare and welfare workers are exposed, for example, to inhalative anesthetics, disinfectants, cleaning agents and surgical fumes.

Objective. The purpose of this study is to determine the extent to which healthcare and welfare workers are aware of their exposure to inhalative noxious agents.

Material and methods. From the baseline sample $(n=10,000)$ of the Hamburg City Health Study (HCHS), all those subjects were selected who were employed in the healthcare and welfare services. Subjective questionnaire data on workplace-related exposure to fumes, gases, dusts and vapors were matched with the categorization generated by a jobexposure matrix (JEM).

Results. The sample $(N=1176)$ included $78 \%$ women, with a mean age of 61 years. Subjects employed in physician and practice assistance (60.5\%) made up the largest occupational group. The agreement between subjective exposure data and the JEM was low (Cohen's kappa 0.18). The sensitivity of the subjective data on inhalative noxious agents in comparison to the JEM was $49.5 \%$, and the specificity was $81.7 \%$. Underreporting of the inhalation of noxious agents was only observed among employees in cleaning and body care.

Discussion. The results indicate that $50 \%$ of exposed workers in this sample are unaware of this exposure. This applies especially to workers in cleaning and personal care. The underreporting of inhalative noxious agents in the workplace indicates an increased need for prevention in this industry. Since there are already existing rules and regulations for the occupational health and safety system, it remains to be clarified to what extent knowledge transfer and prevention rules regarding inhalative noxious agents at the workplace are actually implemented in companies.

\section{Keywords} Occupational $\cdot$ Airborne pollutants $\cdot$ Sensitivity and specificity - Prevention · Health and welfare services angaben zum Beruf ein Berufscode der KldB 2010 zugeordnet werden. Anhand einer Restriktion auf Berufe des Gesundheitsdienstes und der Wohlfahrtspflege ergab sich eine Branchenstichprobe von 1176 (14,4\%) Probanden.

Von diesen waren $77,9 \%$ weiblich und $22,1 \%$ männlich. Das mittlere Alter betrug zum Befragungszeitpunkt 60,8 Jahre, wobei die Männer gering- fügig älter sind (62,2 vs. 60,4 Jahre; $p<0,001$; - Tab. 1). $85,4 \%$ gaben an, in Deutschland geboren $\mathrm{zu}$ sein, für Männer ist dieser Anteil etwas höher $(89,8 \%$ vs. $84,2 \%$; $p=0,024)$. Bezüglich der Schulbildung zeigt sich, dass der größte Teil einen hohen Schulabschluss angab (53,9\%), für 29,4\% wird ein mittlerer und für $14,8 \%$ ein niedriger Schulabschluss beobachtet. Der Anteil der Erwerbstätigen in der Stichprobe liegt bei 69,4\%, wobei sich die Anteile im Geschlechtervergleich kaum voneinander unterscheiden (weiblich: 68,9\%, männlich: 71,2\%).

In - Tab. 2 sind die Häufigkeiten der im Fragebogen angegebene Exposition gegenüber Dämpfen, Gasen, Stäuben und Rauch angegeben sowie die Häufigkeiten auf Grundlage der JEM, die anhand 
Tab. 2 Exposition (Dämpfe, Gase, Stäube, Rauch) nach Fragebogen (Selbstangabe) und nach JEM im Geschlechtervergleich

\begin{tabular}{|c|c|c|c|c|}
\hline & $\begin{array}{l}\text { Weiblich } \\
(N=916)\end{array}$ & $\begin{array}{l}\text { Männlich } \\
(N=260)\end{array}$ & $\begin{array}{l}\text { Gesamt } \\
(N=1176)\end{array}$ & $\begin{array}{l}p- \\
\text { Wert }^{\mathrm{a}}\end{array}$ \\
\hline \multicolumn{4}{|l|}{ Exponiert nach Fragebogen } & $<0,001$ \\
\hline Nicht exponiert & $718(81,7 \%)$ & $180(70,6 \%)$ & $898(79,9 \%)$ & - \\
\hline Exponiert & $161(18,3 \%)$ & $75(29,4 \%)$ & $236(20,1 \%)$ & - \\
\hline$n$ fehlend & 37 & 5 & 42 & - \\
\hline \multicolumn{4}{|c|}{ Expositionskategorisierung I nach JEM } & 0,045 \\
\hline Nicht exponiert & $427(46,6 \%)$ & $103(39,6 \%)$ & $530(45,1 \%)$ & - \\
\hline Wenig/mittel/hoch exponiert & $489(53,4 \%)$ & $157(60,4 \%)$ & $646(54,9 \%)$ & - \\
\hline \multicolumn{4}{|c|}{ Expositionskategorisierung II nach JEM } & 0,081 \\
\hline Nicht/wenig exponiert & $832(90,8 \%)$ & $245(94,2 \%)$ & $1077(91,6 \%)$ & - \\
\hline Mittel/hoch exponiert & $84(9,2 \%)$ & $15(5,8 \%)$ & $99(8,4 \%)$ & - \\
\hline \multicolumn{5}{|l|}{ aPearson's Chi²-Test } \\
\hline
\end{tabular}

der Berufsgruppe zugeordnet werden konnten. Den subjektiven Angaben zufolge zeigt sich ein Expositionsanteil von 20,1\%, wobei der Anteil bei den Männern größer ist als bei den Frauen $(29,4 \%$ vs. $18,3 \% ; p<0,001)$. Bei der Expositionskategorisierung I zeigt sich eine Exposition für die Gesamtgruppe von $54,9 \%$. Im Geschlechtervergleich ist dieser Anteil für Männer höher als für Frauen (60,4\% vs. 53,4\%; $p=0,045)$. Nach den Daten der Expositionskategorisierung II der JEM hingegen ist der Expositionsanteil weitaus geringer als im Vergleich zu den Fragebogenangaben. Für die Gesamtgruppe wird ein Anteil von $8,4 \%$ beobachtet, für die Frauen wird hier allerdings ein höherer Anteil beobachtet als für die Männer (9,2\% vs. $5,8 \% ; p=0,081$ ).

Die Anteile der verschiedenen Berufsgruppen in der Stichprobe zeigt - Tab. 3. Diese Einteilung erfolgte anhand der KldB 2010 Klassifikation auf der Ebene der 3-stelligen Kennzeichnung des 5-stelligen Berufscodes. Beschäftigte aus der Arzt- und Praxishilfe machen dabei mit $60,5 \%$ den größten Anteil der Stichprobe aus, Tierärzte den geringsten $(0,4 \%)$. Die ersten beiden Ergebnisspalten präsentieren die Übereinstimmungen von JEM (Expositionskategorisierung II) und den Fragebogenangaben und die letzten beiden Spalten die Nichtübereinstimmungen. So sind für $4 \%$ eine Übereinstimmung einer Exposition (richtigpositiv) und für $75,1 \%$ eine Übereinstimmung für eine nicht vorhandene Exposition (richtig-negativ) $\mathrm{zu}$ beob- achten. Der Anteil von Probanden mit einer nach JEM nicht vorhandenen und laut Fragebogen vorhandenen Exposition beträgt 16,8\% (falsch-positiv); der Anteil mit einer nach JEM vorhandenen und nach Fragebogen nicht vorhandenen Exposition beträgt 4,1\% (falschnegativ). Diese relevante Risikogruppe (JEM+, FB-) setzt sich aus zwei Berufsgruppen zusammen: Beschäftigte in der Reinigung $(53,1 \%)$ sowie in der Körperpflege (27,3\%).

Bei der Gegenüberstellung der Fragebogenangaben und der JEM (Expositionskategorisierung II) ergibt sich eine Sensitivität von 49,5\% und eine Spezifität von $81,7 \%$. Der berechnete Übereinstimmungskoeffizient Cohens Kappa beträgt $0,18(p<0,001)$. Bei dem Vergleich der Fragebogenangaben mit der JEM nach der Expositionskategorisierung I ergibt sich eine Sensitivität von $28,8 \%$ und eine Spezifität von $88,7 \%$. Der Übereinstimmungskoeffizient Cohens Kappa beträgt $0,17(p<0,001)$.

\section{Diskussion}

In einem Vergleich von subjektiven Expositionsangaben inhalativer Noxen mit den Informationen einer JEM wird in dieser Untersuchung bei Beschäftigten im Gesundheitsdienst und der Wohlfahrtspflege eine Sensitivität von 49,5\% beobachtet (JEM-Expositionskategorisierung II). Dies bedeutet, dass ein Anteil von 50,5\% der nach JEM Exponierten sich dieser Exposition nicht bewusst ist. Die Spezifität lag bei $81,7 \%$, das heißt ein
Anteil von 18,3\% der Nichtexponierten gab an, exponiert zu sein.

Die Subgruppe der bevölkerungsbezogenen Stichprobe der HCHS weist mit einem Branchenanteil von 14,4\% an der Gesamtkohorte eine gute Vergleichbarkeit mit Daten der branchenbezogenen Statistik in Hamburg auf. Die Hamburger Berufsstatistik berichtet für das Jahr 2017 einen Anteil von 11,2\% für die Branche Gesundheits- und Sozialwesen, wobei die Gruppe der Erziehung und Sozialarbeit in diesem Anteil nicht enthalten ist [18]. Der überwiegende Anteil an Frauen von $78 \%$ entspricht der bekannten Geschlechterverteilung dieser Branche. Das Durchschnittsalter von über 60 Jahren lässt sich durch das in der HCHS angewandte Alterseinschlusskriterium (45-74 Jahre) erklären, wobei sich die beobachtete Spannweite von 46-78 Jahren durch eine verzögerte Datenerhebung nach der Randomisierung ergab.

Obwohl die Prävalenz der anhand des Fragebogens erhobenen Exposition weitaus höher lag als bei den JEMDaten (20\% vs. $8 \%$ ), zeigt die Analyse ein Underreporting der Exponierten, welches sich in der geringen Sensitivität von $49,5 \%$ ausdrückt. In vergleichbaren Studien Beschäftigter aller Branchen $\mathrm{zu}$ inhalativen Noxen wurde eine Sensitivität von $42-48 \%, 71 \%$ und $69 \%$ beobachtet [5, 10, 25]. Die Spezifität in diesen Studien lag zwischen 83 und $87 \%, 66 \%$ und $88 \%$, ein Bereich, in den sich die Spezifität aus der vorliegenden Untersuchung von $81,7 \%$ gut einreihen lässt. Bei einem Kollektiv von Beschäftigten im Gesundheitswesen beobachten Delclos et al. im Vergleich zu dieser Untersuchung eine höhere Sensitivität von $67-74 \%$ und eine geringere Spezifität zwischen 64 und $70 \%$ [11]. Cohens Kappa von 0,18 in der vorliegenden Studie weist auf eine geringe Übereinstimmung hin; die Übereinstimmungskoeffizienten der obigen Vergleichsstudien liegen im geringen bis mittleren Bereich von $0,17-0,35,0,35,0,58$ sowie $0,07-0,48$.

Neben der Studie von Delclos et al. [11], welche die Frage zum Expositionswissen bei Beschäftigten im Gesundheitswesen in den USA untersucht, ist unseres Wissens die vorliegende Ar- 


\begin{tabular}{|c|c|c|c|c|}
\hline & $\begin{array}{l}\text { JEM }+ \\
\text { FB }+ \\
\text { Richtig-positiv }\end{array}$ & $\begin{array}{l}\text { JEM- } \\
\text { FB - } \\
\text { Richtig-negativ }\end{array}$ & $\begin{array}{l}\text { JEM - } \\
\text { FB }+ \\
\text { Falsch-positiv }\end{array}$ & $\begin{array}{l}\text { JEM + } \\
\text { FB - } \\
\text { Falsch-negativ }\end{array}$ \\
\hline Berufsgruppen (nach KIdB 3-Steller-Code) & \multicolumn{4}{|c|}{$N=1134(100 \%)$, fehlende Werte: $N=42$} \\
\hline $\begin{array}{l}\text { Arzt- und Praxishilfe }(60,5 \%) \\
\text { (med./zahnmed./tiermed. Fachangestellte, Podologen) }\end{array}$ & $0(0,0 \%)$ & $62(84,9 \%)$ & $11(15,1 \%)$ & $0(0,0 \%)$ \\
\hline $\begin{array}{l}\text { Medizinisches Labor (3,0\%) } \\
\text { (Fachassistenz/Mediziner) }\end{array}$ & $0(0,0 \%)$ & $15(45,5 \%)$ & $18(54,5 \%)$ & $0(0,0 \%)$ \\
\hline Krankenpflege, Rettungsdienst Geburtshilfe (14,8\%) & $0(0,0 \%)$ & $124(73,8 \%)$ & $44(26,2 \%)$ & $0(0,0 \%)$ \\
\hline $\begin{array}{l}\text { Mediziner (13,2\%) } \\
\text { (Fach-, Zahn- und Allgemeinmediziner) }\end{array}$ & $0(0,0 \%)$ & $123(79,9 \%)$ & $31(20,1 \%)$ & $0(0,0 \%)$ \\
\hline Tierärzte/Tierheilpraktiker (0,4\%) & $0(0,0 \%)$ & $3(75,0 \%)$ & $1(25,0 \%)$ & $0(0,0 \%)$ \\
\hline Psychologen $(5,2 \%)$ & $0(0,0 \%)$ & $54(90,0 \%)$ & $6(10,0 \%)$ & $0(0,0 \%)$ \\
\hline $\begin{array}{l}\text { Nichtärztliche Therapie und Heilkunde }(6,4 \%) \\
\text { (Masseur, Physiotherapeut, Logopädie, Heilpraktiker, Gesundheitswissen- } \\
\text { schaftler) }\end{array}$ & $0(0,0 \%)$ & $65(87,8 \%)$ & $9(12,2 \%)$ & $0(0,0 \%)$ \\
\hline $\begin{array}{l}\text { Pharmazie }(4,1 \%) \\
\text { (Apotheker, Pharmakologe, PTA, Pharmareferent, klinischer Monitor) }\end{array}$ & $0(0,0 \%)$ & $33(71,7 \%)$ & $13(28,3 \%)$ & $0(0,0 \%)$ \\
\hline Altenpflege $(7,7 \%)$ & $0(0,0 \%)$ & $65(79,3 \%)$ & $17(20,7 \%)$ & $0(0,0 \%)$ \\
\hline $\begin{array}{l}\text { Berufe in der Gesundheitsberatung }(1,0 \%) \\
\text { (Gesundheitscoach, Ernährungsberater, Ökotrophologe) }\end{array}$ & $0(0,0 \%)$ & $9(75,0 \%)$ & $3(25,0 \%)$ & $0(0,0 \%)$ \\
\hline $\begin{array}{l}\text { Körperpflege ( } 4,1 \%) \\
\text { (Friseur, Kosmetiker, Tätowierer) }\end{array}$ & $23(52,3 \%)$ & $8(18,2 \%)$ & $1(2,3 \%)$ & $12(27,3 \%)$ \\
\hline $\begin{array}{l}\text { Erziehung, Sozialarbeit }(24,5 \%) \\
\text { (Erzieher, Sozialarbeiter, Heilerziehungspfleger, Sozialhelfer, Drogenbera- } \\
\text { ter) }\end{array}$ & $0(0,0 \%)$ & $255(90,1 \%)$ & $28(9,9 \%)$ & $0(0,0 \%)$ \\
\hline Hauswirtschaft $(3,3 \%)$ & $0(0,0 \%)$ & $31(83,8 \%)$ & $6(16,2 \%)$ & $0(0,0 \%)$ \\
\hline $\begin{array}{l}\text { Reinigung ( } 6,0 \%) \\
\text { (Schädlingsbekämpfer, Raumpfleger, Gebäudereiniger, Textilreinigung, } \\
\text { Fahrzeugreiniger) }\end{array}$ & $22(34,4 \%)$ & $5(7,8 \%)$ & $3(4,7 \%)$ & $34(53,1 \%)$ \\
\hline Gesamt & $45(4,0 \%)$ & $852(75,1 \%)$ & $191(16,8 \%)$ & $46(4,1 \%)$ \\
\hline
\end{tabular}

beit die einzige deutsche Untersuchung bei Beschäftigten im Gesundheitsdienst und der Wohlfahrtspflege zu diesem Thema. Der Vergleich der Analysen nach JEM-Expositionskategorisierung I und II zeigt insbesondere hinsichtlich der Sensitivität, die aus präventiver Sicht das wichtigere Beurteilungskriterium ist, einen erheblichen Unterschied. Bei dem Einschluss der wenig Exponierten in die Expositionsklasse (Expositionskategorisierung I), würde sich die Sensitivität fast halbieren ( $28,8 \%$ vs. $49,5 \%)$. Das heißt, der Anteil der Exponierten, die sich ihrer Exposition nicht bewusst sind, würde sich von $50,5 \%$ auf $71,2 \%$ erhöhen. Um einer fehlerhaften Zuordnung von laut JEM wenig Exponierten zu der Exponiertenklasse vorzubeugen und ohne Einbeziehung der Angaben, welche Häufigkeiten je Expositionsart hinter der übergeordneten Frage stehen, favo- risieren wir eine eher konservative und moderate Interpretation der Ergebnisse. Aus diesen Gründen wie auch aufgrund der oben erwähnten übereinstimmenden Daten zu der Sensitivität aus Vergleichsstudien ziehen wir für die Analyse die Expositionskategorisierung II vor.

In einer Arbeit von Kurth et al. zeigt sich hinsichtlich der Validität von JEMDaten im Vergleich zu Expertenbeurteilungen von beruflichen inhalativen Expositionen eine moderate Übereinstimmung (Cohens Kappa: 0,51) sowie eine Sensitivität von $68 \%$ und eine Spezifität von $83 \%$ [22]. Unterstellt man daher in der vorliegenden Untersuchung den JEM-Daten im Vergleich zu den subjektiven Expositionsangaben eine höhere Validität, zeigt sich in diesem Kollektiv ein Präventionsbedarf hinsichtlich inhalativer Noxen am Arbeitsplatz. Dies gilt insbesondere für Beschäftigte in der Rei- nigung und der Körperpflege. Bei Arbeiten mit Desinfektions- und Reinigungsmittel kommt es z. B. zu Dampfexpositionen mit Formaldehyd, Glutaraldehyd oder 2-Butoxyethanol, welche akut toxisch wirken bzw. zu einer Sensibilisierung der Atemwege und zu einer Einschränkung der Lungenfunktion führen können [12, 27]. Im Friseurgewerbe kann es aufgrund von z.B. Tönungsmitteln, Fixiermitteln und Haarsprays zu Expositionen von verschiedenen chemischen Stoffen wie Wasserstoffperoxid und Persulfaten kommen, welche Reizungen der Schleimhäute und Sensibilisierungen der Atemwege zur Folge haben können [4, 23].

Um diese Ergebnisse in den Kontext der Prävention zu stellen, sei an dieser Stelle ein begrenzter Einblick in relevante Regelungen im Arbeitsund Gesundheitsschutz und deren be- 


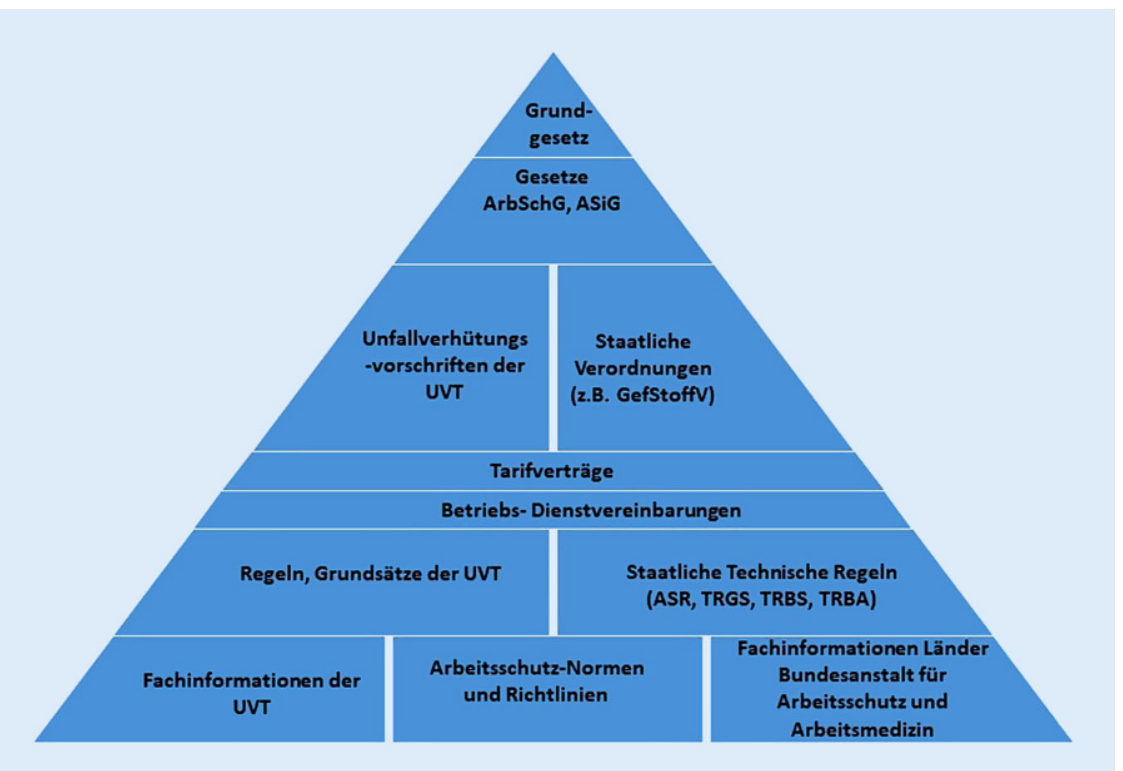

Abb. 1 A Pyramide des Arbeitsschutzrechts. ArbSchG Arbeitsschutzgesetz, ASiG Arbeitssicherheitsgesetz, UVT Unfallversicherungsträger, GefStoffV Gefahrstoffverordnung, ASR Technische Regeln für Arbeitsstätten, TRGS Technische Regeln für Gefahrstoffe, TRBS Technische Regeln für Betriebssicherheit, TRBA Technische Regeln für Biologische Arbeitsstoffe. (Modifizierte Abbildung, Quelle: DGUV, Gestaltung Regine Rundnagel)

triebliche Umsetzung in Bezug auf den Informationsfluss gegeben. Einen Gesamtüberblick über das Arbeitsschutzrecht in Deutschland liefert die Pyramide in $\bullet$ Abb. 1.

Wesentliche Elemente sind die rechtsverbindliche Gefahrstoffverordnung [9] mit den Technischen Regeln für Gefahrstoffe (TRGS) und die Vorschriften der Unfallversicherungsträger mit einer Reihe von Grundsätzen, Regeln und Fachinformationen. Diese sollen die Beschäftigten bzgl. der Exposition inhalativer Noxen schützen. Konkrete Beispiele für die genannten Branchen sind:

- TRGS 402 „Ermitteln und Beurteilen der Gefährdungen bei Tätigkeiten mit Gefahrstoffen: Inhalative Exposition“

- TRBA/TRGS 406 „Sensibilisierende Stoffe für die Atemwege“

- TRGS 500 „Schutzmaßnahmen“

- TRGS 525 „Gefahrstoffe in Einrichtungen der medizinischen Versorgung"

- TRGS 530 „Friseurhandwerk“

Die Regelungen richten sich an die Unternehmensleitung. Sie oder die zuständige Führungskraft sind für den Informationsfluss und die Infrastruktur dieser Maßnahmen vor Ort verantwort-

\section{Limitationen}

Die Verwendung von JEMs birgt ein Verzerrungspotenzial aufgrund von Missklassifikation [24]. So wird allen Beschäftigten einer Berufsgruppe eine bzw. keine Exposition zugewiesen, welches auf Individualebene nicht den Tatsachen entsprechen muss. Aufgrund der geringen Fallzahlen wurden die Berufsgruppen auf die Ebene der 3-Steller-Codes aggregiert. Dies hat zur Folge, dass innerhalb einer aggregierten Gruppe sowohl richtig-positive als auch richtig-negative Exponierte vorzufinden sind. Eine berufsgruppenspezifische Interpretation sollte daher sehr vorsichtig erfolgen und die Interpretation für die Gesamtgruppe Vorrang haben.

Ein Vorteil bei der Verwendung einer JEM besteht hingegen darin, dass für Beschäftigte, die ihre frühere Exposition nicht mehr erinnern (Recall-Bias) oder niemals von ihr erfahren haben, eine von Messungen an einer Auswahl von Arbeitsplätzen abgeleitete Exposition zugeteilt werden kann. Dies trifft auch auf die Expositionshöhe zu, der sie selbst an ihrem Arbeitsplatz ausgesetzt waren und oftmals unzureichend einschätzen können. Um die Expositionszuweisung nicht zu überschätzen, erfolgte eine konservative Zuordnung anhand der Expositionshöhe von Exponierten und Nichtexponierten.

\section{Schlussfolgerungen}

Es gibt Hinweise, dass insbesondere bei Klein- und Mittelbetrieben die Durchführung von betrieblichen Gefährdungsbeurteilungen problematisch ist [16]. Die Unfallversicherungsträger/BGW und die staatlichen Aufsichtsbehörden fungieren als Beratungs- und Kontrollorgane. Es erweist sich jedoch allein schon aufgrund der Vielzahl der Betriebe als schwierig, bei jenen, geschweige denn allen Betrieben diese Funktion auszuüben. Eine Überprüfung der (betrieblichen) Umsetzung der Anforderungen aus den Gefahrstoffregelungen von extern ist somit nur bedingt möglich.
Das in dieser Studie beobachtete Underreporting gegenüber inhalativen Noxen weist für Beschäftigte im Gesundheitsdienst und der Wohlfahrtspflege, speziell für Beschäftigte in der Reinigung und der Körperpflege, einen offensichtlichen Präventionsbedarf auf. Demgegenüber stehen die Vorschriften und Regeln des Arbeitsschutzsystems, über deren Umsetzung bislang noch keine Datenbasis existiert. Es bleibt zu untersuchen, inwieweit die verfassten Präventionsregeln tatsächlich in Betrieben umgesetzt werden. Das alleinige Vorhandensein von Vorschriften und Regeln vor Ort sichert nicht unbedingt die Wissensverbreitung und die Umsetzung der Präventionsprinzipien. Um die beobachteten Ergebnisse bes- 
ser interpretieren zu können, sollten betriebsbezogene Untersuchungen zu dieser Thematik durchgeführt werden. So könnten weitere Erkenntnisse zu dem Funktionieren der Präventionskette bei inhalativen Noxen generiert werden.

\section{Fazit für die Praxis}

\section{- Der Befund dieser Datenanalyse gibt den Hinweis, dass ein Teil der Beschäftigten aus dem Gesundheits- dienst und der Wohlfahrtspflege (Beschäftigte aus der Reinigung und der Körperpflege) sich ihrer Expo- sition gegenüber Dämpfen, Gasen, Stäuben und Rauch nicht bewusst sein könnte. \\ - Es existiert ein umfassendes Re- gelwerk des Arbeitsschutzsystems, welches die Information über die Existenz dieser Gefahrstoffe wie auch die Präventionsstrategien zur Kontaktreduzierung regelt. \\ - Es existiert eine Forschungslücke, inwieweit die Informations- und Präventionsstrategien des Arbeits- schutzsystems hinsichtlich inha- lativer Noxen am Arbeitsplatz auf Betriebsebene mit den Beschäftigten umgesetzt werden. \\ - Untersuchungen dazu könnten zu der Wirkungsevaluation der vorhandenen Präventionsstrategien beitragen.}

\section{Korrespondenzadresse}

\section{Dr. Peter Koch}

Institut für Versorgungsforschung in der Dermatologie und bei Pflegeberufen, Universitätsklinikum Hamburg-Eppendorf (UKE)

Martinistr. 52, 20246 Hamburg, Deutschland p.koch@uke.de

Funding. Open Access funding enabled and organized by Projekt DEAL.

\section{Einhaltung ethischer Richtlinien}

Interessenkonflikt. P. Koch, G. Halsen, L. Damerau, A. Nienhaus, V. Harth, M. Augustin und C. Terschüren geben an, dass kein Interessenkonflikt besteht.

Für diesen Beitrag wurden von den Autoren keine Studien an Menschen oder Tieren durchgeführt. Für die aufgeführten Studien gelten die jeweils dort angegebenen ethischen Richtlinien.
Open Access. Dieser Artikel wird unter der Creative Commons Namensnennung 4.0 International Lizenz veröffentlicht, welche die Nutzung, Vervielfältigung, Bearbeitung, Verbreitung und Wiedergabe in jeglichem Medium und Format erlaubt, sofern Sie den/die ursprünglichen Autor(en) und die Quelle ordnungsgemäß nennen, einen Link zur Creative Commons Lizenz beifügen und angeben, ob Änderungen vorgenommen wurden.

Die in diesem Artikel enthaltenen Bilder und sonstiges Drittmaterial unterliegen ebenfalls der genannten Creative Commons Lizenz, sofern sich aus der Abbildungslegende nichts anderes ergibt. Sofern das betreffende Material nicht unter der genannten Creative Commons Lizenz steht und die betreffende Handlung nicht nach gesetzlichen Vorschriften erlaubt ist, ist für die oben aufgeführten Weiterverwendungen des Materials die Einwilligung des jeweiligen Rechteinhabers einzuholen.

Weitere Details zur Lizenz entnehmen Sie bitte der Lizenzinformation auf http://creativecommons.org/ licenses/by/4.0/deed.de.

\section{Literatur}

1. Balmes J, Becklake M, BlancPet al (2003) American Thoracic Society Statement: occupational contribution to the burden of airway disease. Am J Respir Crit Care Med 167:787-797

2. Barone-Adesi F, Richiardi L, Merletti F (2005) Population attributable risk for occupational gancer in Italy. Int J Occup Environ Health 11:23-31

3. Baur X, Sigsgaard T, Aasen T et al (2012) Guidelines for the management of work-related asthma. Eur Respiratory Soc 39:529-545

4. Berufsgenossenschaft für Gesundheitsdienst und Wohlfahrtspflege (BGW) (2020) Betriebsanweisung für Friseurinnen und Friseure. https://www. bgw-online.de/DE/Medien-Service/MedienCenter/Medientypen/BGW-Broschueren/ BGW09-19-091_Betriebsanweisung-Friseurhand werk.html.Zugegriffen:08.09.2021

5. Blanc PD, Annesi-Maesano I, Balmes JR et al (2019) The occupational burden of nonmalignant respiratory diseases. An official American Thoracic Society and European Respiratory Society statement. Am J Respir Crit Care Med 199:1312-1334

6. Bundesagentur für Arbeit (2011a) Systematischer und alphabetischer Teil mit Erläuterungen. Klassifikation der Berufe 2010 (KIdB 2010), Bd. I

7. Bundesanstalt für Arbeitsschutz und Arbeitsmedizin (2006) Technische Regeln für Gefahrstoffe (TRGS). https://www.baua.de/DE/Angebote/ Rechtstexte-und-Technische-Regeln/Regelwerk/ TRGS/TRGS.html.. Zugegriffen:08.09.2021

8. Bundesanstalt für Arbeitsschutz und Arbeitsmedizin (2012) Technische Regeln für Arbeitsschutz: ASR A3.6 Lüftung. https://www.baua.de/DE/ Angebote/Rechtstexte-und-Technische-Regeln/ Regelwerk/ASR/ASR-A3-6.html.. Zugegriffen: 08.09.2021

9. Bundesministerium der Justiz und für Verbraucherschutz (2010) Verordnung zum Schutz vor Gefahren. https://www.gesetze-im-internet.de/ gefstoffv_2010/..Zugegriffen:08.08.2021

10. De Vocht F, Zock JP, Kromhout H et al (2005) Comparison of self-reported occupational exposure with a job exposure matrix in an international community-based study on asthma. Am J Ind Med 47:434-442
11. Delclos GL, Gimeno D, Arif AA et al (2009) Occupational exposures and asthma in health-care workers: comparison of self-reports with a workplace-specific job exposure matrix. Epidemiol Rev 169:581-587

12. Deutsche Gesetzliche Unfallversicherung (DGUV) (2021) DGUV Information Gefahrstoffe im Gesundheitsdienst. https://publikationen. dguv.de/regelwerk/dguv-informationen/844/ gefahrstoffe-im-gesundheitsdienst.. Zugegriffen: 08.09.2021

13. Dumas O, Varraso R, Boggs KM et al (2019) Association of occupational exposure to disinfectants with incidence of chronic obstructive pulmonary disease among US female nurses. JAMA Netw Open 2:e1913563-e1913563

14. Elias P, Mcknight A, Kinshott G (1999) SOC 2000: redefining skill: revision of the standard occupational classification

15. Ganzeboom HB (2010) International standard classification of occupations ISCO-08 with ISEI-08 scores (Version of July 27:2010)

16. Hinsch J, Nienhaus A (2014) Gefärdungsbeurteilung in Kleinbetrieben am Beispiel von Tageseinrichtungen für Kinder. In: Nienhaus A (Hrsg) RiRe - Risiken und Ressourcen im Gesundheitsdienst und der Wohlfahrtspflege. ecomed MEDIZIN, Heidelberg, S 180-202

17. Hnizdo E, Vallyathan V (2003) Chronic obstructive pulmonary disease due to occupational exposure to silica dust: a review of epidemiological and pathological evidence. Occup Environ Med 60:237-243

18. Institutfür Arbeitsmarktund Berufsforschung (IAB) (2018) Berufe im Spiegel der Statistik. http://bisds. iab.de/Default.aspx?beruf $=A B O \&$ region $=7$ \& qualifikation $=0$..Zugegriffen: 08.09.2021

19. Jagodzinski A, Johansen C, Koch-Gromus U et al (2020) Rationale and design of the Hamburg City Health study. Eur JEpidemiol 35:169-181

20. Jöckel K, Ahrens W, Bolm-Audorff $U$ et al (1997) Occupationally-induced lung cancer-A quantitative evaluation for the North Germany area. Gesundheitswesen 59:275-278

21. Kogevinas M, ZockJ-P, Jarvis Detal (2007) Exposure to substances in the workplace and new-onset asthma: an international prospective populationbased study (ECRHS-II). Lancet 370:336-341

22. Kurth L, Doney B, Weinmann S (2017) Occupational exposures and chronic obstructive pulmonary disease (COPD): comparison of a COPD-specific job exposure matrix and expert-evaluated occupational exposures. Occup Environ Med 74:290-293

23. Moscato G, Galdi E (2006) Asthma and hairdressers. Curr Opin Allergy Clin Immunol 6:91-95

24. Olsen J (1988) Limitations in the use of job exposure matrices. Scand J Soc Med 16:205-208

25. Quinlan PJ, Earnest G, Eisner MD et al (2009) Performance of self-reported occupational exposure compared to a job-exposure matrix approach in asthma and chronic rhinitis. Occup Environ Med 66:154-160

26. Sadhra S, Kurmi OP, Sadhra SS et al (2017) Occupational COPD and job exposure matrices: a systematic review and meta-analysis. Int J Chron Obstruct Pulmon Dis 12:725

27. Svanes $\emptyset$, Bertelsen RJ, Lygre SH et al (2018) Cleaning at home and at work in relation to lung function decline and airway obstruction. Am J Respir Crit Care Med 197:1157-1163

28. Tarlo SM, Lemiere C (2014) Occupational asthma. NEngl J Med 370:640-649 